routine internal examination deters many of the patients we should most like to see at the postnatal clinic. They avoid the doctor by attending the local authority clinic. Assuming a normal history, as already outlined, then I am not at all convinced of the value of a vaginal examination. What treatable condition am I supposed to discover? My limited experience leads me to believe my time would be more usefully spent pursuing some of my other obligations to the mother and child.

(2) I check her weight and give advice if necessary.

(3) I check her blood pressure and act accordingly.

(4) I take a routine venous specimen of blood for haemoglobin estimation.

This routine specimen of blood could be criticized on the grounds that it causes unnecessary pain to the patient; the local laboratory receive yet more quantitative estimations to perform; and that I should use my clinical judgement and do a haemoglobin on those "at risk"-that is, social class IV, those that lost above average amounts of blood during labour, and those with borderline haemoglobin prior to confinement (this is checked on first visit and at 32 weeks).

I would reply that I do not trust my eyes as far as haemoglobin is concerned, and that having discovered anaemia I can at least do something about it.

I do not examine urine routinely at the postnatal consultation (I do so at each consultation during pregnancy), but I do ask for a specimen of urine if there is any history of albuminuria.

(5) We discuss contraception and family planning. This often takes more time than the previous four procedures together - and deservedly so.

The ideal is seldom achieved and I would be grateful for commerts on these routine measures. Are we concentrating on those things that matter most or are we spending time carrying out procedures motivated by teaching and habits rather than critical assessment of what is of greatest long-term value to the mother and child ?-I am, etc.,

$$
\text { Middlesbrough. IAN CAPSTICK. }
$$

\section{Aphthous Ulceration}

SIR,-As the aetiology and effective therapy for this common condition remain problematical, may I record experiences both as patient and physician.

Suffering from very extensive and recurrent aphthous ulceration as a student, I sought the advice of the late Mr. Cobb, of Sheffield, well before the era of antibiotics. He prescribed a mouthwash of a saturated fresh solution of chlorate of potash with the addition of a little phenol and glycerin, to be used before and after meals as well as night and morning, emphasizing that old solutions were valueless, as are tablets.

The treatment was rapidly effective and has been of great value to many patients, aborting attacks if used early and frequently enough.

The early lesion in the mucous membrane has the appearance of a small blood blister. Once it breaks down it rapidly extends into a painful ulcer, presumably by bacterial colonization.

I believe that freshly made solutions frequently applied, and of course as early as possible, will still be found to be very efficacious. - I am, etc.,

S. Howard Ferguson.

Florida State Board of Health,

Jacksonvil!e, U.S.A.

\section{Price of Blood}

SIR,-We agree with Dr. A. J. Zuckerman's arguments (20 April, p. 174) against the payment of blood donors, but we wish to draw attention to our own recent survey ${ }^{1}$ of the incidence of anicteric hepatitis following blood transfusion in Birmingham. No case was found among 42 patients who received 128 pints of blood, suggesting that the incidence in this country may indeed be much lower than in the countries cited by $\mathrm{Dr}$. Zuckerman.

We were unable to determine whether this relative immunity was due to a low incidence of viral hepatitis in the community or to the simple methods used to exclude donors who might be potential carriers. We feel, however, that it would be extremely unwise to adopt a system which has been shown to attract donors who display an increased incidence of abnormal liver-function tests, ${ }^{2}$ and whose blood gives rise to serum hepatitis more often than that given by volunteers. ${ }^{3}-$ We are, etc.,

\section{Dudley Road Hospital. B. N. SOMAYAJr. Birmingham 18.}

\section{REFERENCES}

Somayaji, B. N., Stone, W. D., and Glover, P.. B., Gut, 1967, 8, 614. J. G., Transfusion (Philad.), $1963,3,202$. Grady, G. F. Chalmers, T. C., and the Boston
Inter-Hospital Liver Group, New Engl. $f$. Inter-Hospital Liver
Med., 1964, 271, 337.

\section{Overcrowding in Psychiatric Hospitals}

SIR,-In replying to my letter (13 April, p. 116) with regard to overcrowding in psychiatric hospitals, Dr. Martin Davies (27 April, p. 240) discusses the problem of community care. Surely the community doctor is the general practitioner? The hospital doctor should not be going out doing the general practitioner's work for him. If this is done an expensive duplication of care will follow.

I do not think that Dr. J. C. Barker (4 May, p. 300) should be unduly concerned about "revolving doors" at Rubery. Surely these are preferable to locked closed doors, and psychiatry is in the main concerned with recurrent illnesses. The patient should be kept in the community as much as possible and there follows an escalation into better mental health. This has been noted time and time again at Rubery Hill Hospital. And Dr. Barker is surely using wrong techniques in handling a large long-stay population by individual interview methods. He should be employing ward conference and group review methods and not the cumbersome time-consuming individual interview. Group methods have many therapeutic benefits, as has been shown in the development of therapeutic communities. At Rubery Hill in my own unit every ward is using ward conference and case conference review techniques with full use of group meetings. These methods have enabled every patient in the unit's 12 wards to be under full review and close medical supervision by the consultant. The results I described in my letter. I would add that in the last six years only 35 patients have become long-stay patients, and all of these have organic dementias. This is out of a total of about 3,500 admissions during this period.
One can only view with grave concern the practice of locking hospital patients into wards. There is absolutely no need for this dangerous obnoxious practice. In my own unit at Rubery Hill Hospital, which mainly comprises the male half of the hospital, there is not a single locked door.

The usual pleas for more and more staff have been made. It is my view that what is needed is better deployment of medical staff and more energy and enthusiasm on the part of consultants, who should mainly concern themselves with active patient management and care. It must be remembered that half of all hospital beds in this country are occupied by psychiatric cases. The general use of techniques now fully employed at Rubery Hill Hospital would save the National Health Service hundreds of millions of pounds every year and would rapidly produce an excess of hospital beds throughout the country.-I am, etc.,

\section{Rubery Hill Hospital, Birningham.}

Charles Entwistle.

\section{Design for Ambulances}

SIR,-Mr. P. S. London (see leading article, 11 May, p. 318) and Dr. P. W. Bothwell (11 May, p. 366) it is hoped will have helped in their respective spheres to achieve the construction of an ambulance worthy of our era. We all know what "shocking" things they are now.

What is needed is not a better vehicle, but a designed unit installed in a vehicle, be it a Daimler suspension, a car (car ambulance), or a van. This unit should be installed as easily and cheaply as possible, and it should eliminate vibration, sway, shock, set, and drift, and have associated wide load range. Also incorporated should be adjustment for level travelling or head up or down position.

This is not a car manufacturer's problem or such a big problem as it seems. In fact there are available components to eliminate vibration, etc., but to my knowledge positioning needs some further research to make the unit a fait accompli.-I am, etc.,

Ryde,

Trevan Hambly.

Isle of Wight.

\section{Bacillary Dysentery}

SIR,-It is regrettable that a comprehensive article on bacillary dysentery (4 May, p. 285) should have to be criticized on the grounds of omission. In discussing treatment, no mention is made of colistin sulphate, although a recent paper ${ }^{1}$ reports a $73.3 \%$ success rate in eradicating Shigella sonnei or Sh. flexneri. The criterion used in evaluating these cases was the occurrence of six consecutive negative dismissal swabs. This substantiates previous work $^{2}$ demonstrating the efficacy of colistin sulphate against Sh. sonnei.

While these are encouraging therapeutic results, their importance is more apparent in the light of the findings of Smith and Armour. While studying the phenomenon of extrachromosomal transferable resistance ( $R$ factor) they observed that resistance to the polymyxins (of which colistin is a 